
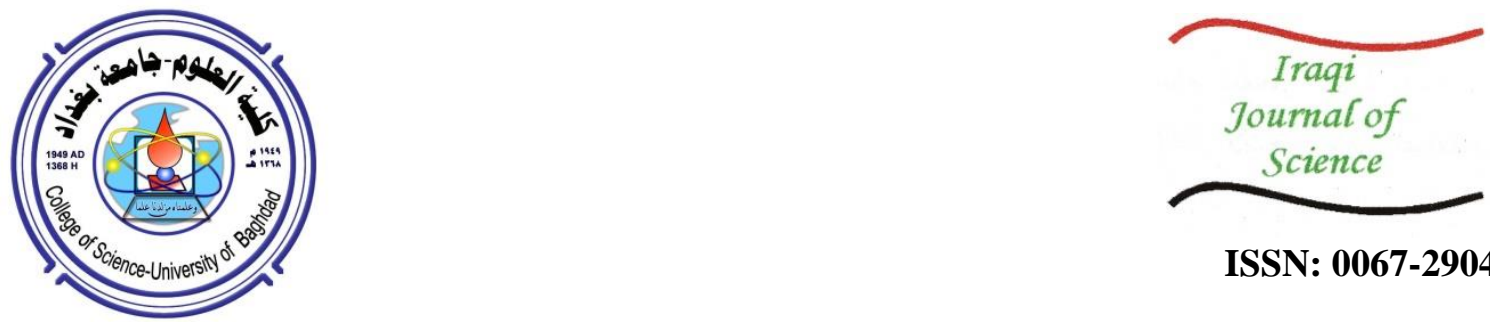

ISSN: 0067-2904

\title{
Some Geometric Properties for a Certain Class of Meromorphic Univalent Functions by Differential Operator
}

\author{
Mohammed Hadi Lafta
}

Department of Business Administration, College Administration and Economics, University of Sumer, Iraq

Received: 9/7/2020

Accepted: 20/11/2020

\begin{abstract}
:
The major target of this paper is to study a confirmed class of meromorphic univalent functions $\mathcal{K}(\varepsilon, \tau, \alpha, \beta, v)$. We procure several results, such as those related to coefficient estimates, distortion and growth theorem, radii of starlikeness, and convexity for this class, $\mathrm{n}$ additionto hadamard product, convex combination, closure theorem, integral operators, and $\sigma$ neighborhoods.
\end{abstract}

Keywords: Meromorphic univalent function, distortion and growth theorem, integral operators, differential operator, convex combination, integral operator and $\sigma$ neighborhoods.

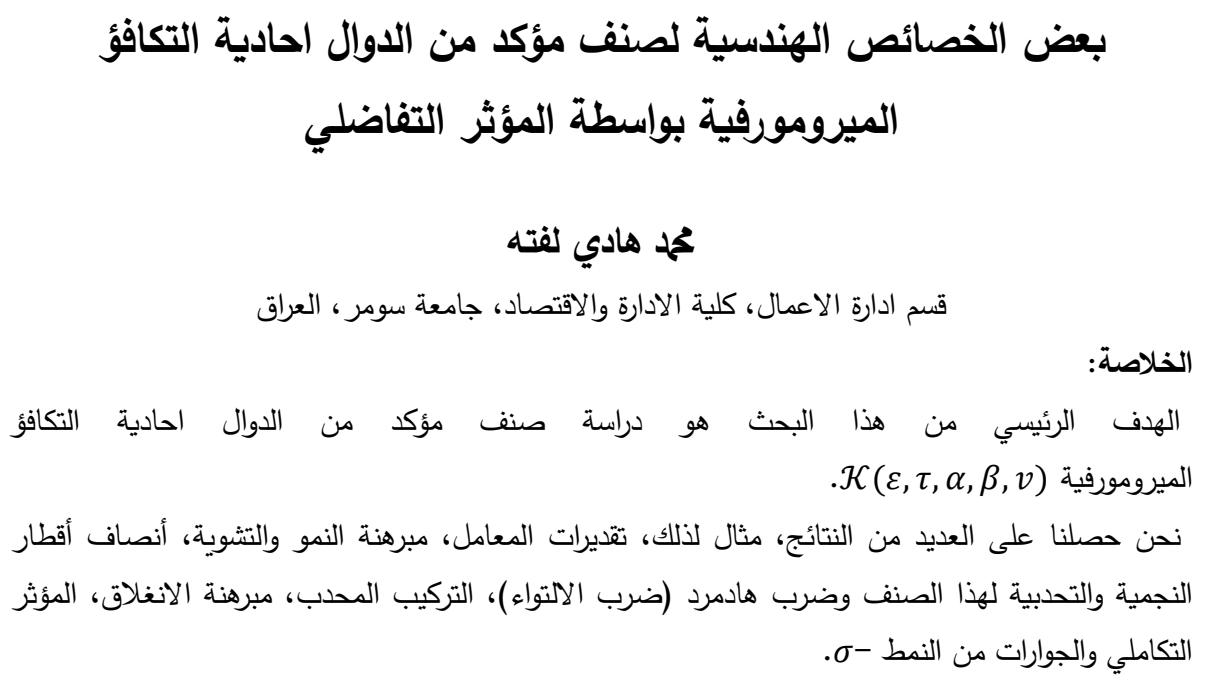

\section{1- Introduction}

We have studied the geometric function theory of the blending of geometry and analysis. Its radix has begun from the 19th century, but it uninterrupted and permanent is applicable at present.

Geometric function theory is a paramount department of complex analysis, which studies the geometric results of the analytic functions.

An univalent function is meromorphiic function $f$ in a domain of expanded complex plane, so that $f\left(z_{1}\right) \neq f\left(z_{2}\right)$ for $z_{1} \neq z_{2}$, where $z_{1}$ and $z_{2}$ are members of the domain.

One of the fundamental troubles in the treatise of univalent functions is whether there exists an univalent mapping of plain linked domain onto a given plain linked domain. Nevertheless, at view of Riemann Mapping theorem [1] over trouble reduces to a trouble of mapping a unit disk onto a given plain linked domain, such as like stairlike, convex, close-to-convex etc.

*Email: mmohamnedhadi@gmail.com 
Let $\mathcal{N}$ denotes the analytic and meromorphic univalent class form functions in the punctured unit disk $U^{*}=\{\mathrm{z} \in \mathbb{C}: 0<|\mathrm{z}|<1\}=U-\{0\}$ and let $\mathcal{A}(j)$ indicates the subclass of $\mathcal{N}$ made up of functions of the form:

$$
f(\mathrm{z})=\mathrm{z}^{-1}-\sum_{j=1}^{\infty} a_{j} \mathrm{z}^{j}, \quad\left(a_{k} \geq 0, j \in \mathbb{N}=\{1,2, \ldots\}\right),
$$

which are analytic functions and meromorphic univalent functions in $U^{*}$.

A function $f \in \mathcal{A}(j)$ is said to be meromorphically starlike of order $\gamma$ if

$$
R e\left\{-\frac{\mathrm{zf}(\mathrm{z})}{f(\mathrm{z})}\right\}>\gamma,\left(\mathrm{z} \in U=U^{*} \cup\{0\}, 0 \leq \gamma<1\right),
$$

and a function $f \in \mathcal{A}(j)$ is said to be meromorphically convex of order $\gamma$ if

$$
\operatorname{Re}\left\{-\left(1+\frac{\mathrm{zf^{ \prime \prime } ( \mathrm { z } )}}{f^{\prime}(\mathrm{z})}\right)\right\}>\gamma,\left(\mathrm{z} \in U=U^{*} \cup\{0\}, 0 \leq \gamma<1\right) .
$$

We indicate by $\delta^{*}(\gamma)$ and $\delta(\gamma)$, respectively, the classes of univalent meromorphiic starlike functions of order $\gamma$ and univalent meromorphic convex functions of order $\gamma$. Such classes have been extensively calculated previously[2-7].

The differential operator $\left(\mathcal{L}_{\alpha, \beta}\right)[8]$ below will be used for this paper:

$$
\begin{aligned}
\mathcal{L}_{\alpha, \beta} f(\mathrm{z})=\mathrm{z}^{-1}- & \sum_{j=1}^{\infty} a_{j}[\alpha(j+2)(\beta(j+1)+1)-\beta(j+2)+(1-\alpha+\beta)] \mathrm{z}^{j} \\
& =\mathrm{z}^{-1}-\sum_{j=1}^{\infty} \mathcal{H}(\alpha, \beta, j) a_{j} \mathrm{z}^{j},
\end{aligned}
$$

such that $\mathcal{H}(\alpha, \beta, j)=[\alpha(j+2)(\beta(j+1)+1)-\beta(j+2)+(1-\alpha+\beta)]$, $\beta \leq \alpha, j \in \mathbb{N}=\{1,2, \ldots\}$.

Definition (1): A function $f \in \mathcal{A}(j)$ is in the class $\mathcal{K}(\varepsilon, \tau, \alpha, \beta, v)$ if the following condition is met:

$$
R e\left\{1+\frac{1}{\tau}\left(1+\frac{\mathrm{z}\left(\mathcal{L}_{\alpha, \beta} \mathrm{f}(\mathrm{z})\right)^{\prime \prime}}{\left(\mathcal{L}_{\alpha, \beta} f(\mathrm{z})\right)^{\prime}}-\varepsilon\right)\right\}>v\left|1+\frac{1}{\tau}\left(\frac{z\left(\mathcal{L}_{\alpha, \beta} \mathrm{f}(\mathrm{z})\right)^{\prime \prime}}{\left(\mathcal{L}_{\alpha, \beta} f(\mathrm{z})\right)^{\prime}}\right)\right|, \quad \mathrm{z} \in U^{*},
$$

such that $-1<v<\varepsilon<1, \tau \in \mathbb{C} \backslash\{0\}$.

\section{2- Coefficient Estimates}

The theorem below provides a sufficient condition for a function in $K(\varepsilon, \tau, \alpha, \beta, v)$.

Theorem (1): A function $f \in \mathcal{A}(j)$ is in the class $\mathcal{K}(\varepsilon, \tau, \alpha, \beta, v)$ if and only if

$$
\sum_{j=1}^{\infty} j[(j+|\tau|)(1-v)+(v-\varepsilon)] \mathcal{H}(\alpha, \beta, j) a_{j} \leq(1-v)(1+|\tau|)+(\varepsilon-v),
$$

such that $-1<v<\varepsilon<1, \tau \in \mathbb{C} \backslash\{0\}$.

The outcome is sharp for the function

$$
f(z)=z^{-1}-\frac{(1-v)(1+|\tau|)+(\varepsilon-v)}{j[(j+|\tau|)(1-v)+(v-\varepsilon)] \mathcal{H}(\alpha, \beta, j)} \mathrm{z}^{j}, \quad j \in \mathbb{N} .
$$

Proof: Suppose that (6) is true for $|z|=1$. Then

if

$$
\operatorname{Re}\left\{1+\frac{1}{\tau}\left(1+\frac{\mathrm{z}\left(\mathcal{L}_{\alpha, \beta} \mathrm{f}(\mathrm{z})\right)^{\prime \prime}}{\left(\mathcal{L}_{\alpha, \beta} \mathrm{f}(\mathrm{z})\right)^{\prime}}-\varepsilon\right)\right\}-v\left|1+\frac{1}{\tau}\left(\frac{\mathrm{z}\left(\mathcal{L}_{\alpha, \beta} \mathrm{f}(\mathrm{z})\right)^{\prime \prime}}{\left(\mathcal{L}_{\alpha, \beta} \mathrm{f}(\mathrm{z})\right)^{\prime}}\right)\right|>0,
$$

that is if

$$
\begin{gathered}
1+\frac{1}{|\tau|}\left(\frac{(1+\varepsilon)-\sum_{j=1}^{\infty} j(j-\varepsilon) \mathcal{H}(\alpha, \beta, j) a_{j}}{-1-\sum_{j=1}^{\infty} j \mathcal{H}(\alpha, \beta, j) a_{j}}\right) \\
-v\left[1+\frac{1}{|\tau|}\left(\frac{2-\sum_{j=1}^{\infty} j(j-1) \mathcal{H}(\alpha, \beta, j) a_{j}}{-1-\sum_{j=1}^{\infty} j \mathcal{H}(\alpha, \beta, j) a_{j}}\right)\right]>0,
\end{gathered}
$$




$$
\sum_{j=1}^{\infty} j[(j+|\tau|)(1-v)+(v-\varepsilon)] \mathcal{H}(\alpha, \beta, j) a_{j} \leq(1-v)(1+|\tau|)+(\varepsilon-v) .
$$

Conversely, presume that $f \in \mathcal{K}(\varepsilon, b, \alpha, \beta, v)$, then

$$
\begin{gathered}
\operatorname{Re}\left\{1+\frac{1}{\tau}\left(1+\frac{\mathrm{z}\left(\mathcal{L}_{\alpha, \beta} f(\mathrm{z})\right)^{\prime \prime}}{\left(\mathcal{L}_{\alpha, \beta} f(\mathrm{z})\right)^{\prime}}-\varepsilon\right)\right\}>v\left|1+\frac{1}{\tau}\left(\frac{\mathrm{z}\left(\mathcal{L}_{\alpha, \beta} f(\mathrm{z})\right)^{\prime}}{\left(\mathcal{L}_{\alpha, \beta} f(\mathrm{z})\right)^{\prime}}\right)\right|, \\
\operatorname{Re}\left\{1+\frac{1}{\tau}\left(\frac{(1+\varepsilon)-\sum_{j=1}^{\infty} j(j-\varepsilon) \mathcal{H}(\alpha, \beta, j) a_{j}}{-1-\sum_{j=1}^{\infty} j \mathcal{j}(\alpha, \beta, j) a_{j}}\right)\right\} \\
>v\left|1+\frac{1}{\tau}\left(\frac{2-\sum_{j=1}^{\infty} j(j-1) \mathcal{H}(\alpha, \beta, j) a_{j}}{-1-\sum_{j=1}^{\infty} j \mathcal{H}(\alpha, \beta, j) a_{j}}\right)\right| .
\end{gathered}
$$

By letting $\mathrm{z} \rightarrow 1^{-}$along the real axis, we get

$$
\begin{aligned}
& 1+\frac{1}{|\tau|}\left(\frac{(1+\varepsilon)-\sum_{j=1}^{\infty} j(j-\varepsilon) \mathcal{H}(\alpha, \beta, j) a_{j}}{-1-\sum_{j=1}^{\infty} j \mathcal{H}(\alpha, \beta, j) a_{j}}\right) \\
& >v\left[1+\frac{1}{|\tau|}\left(\frac{2-\sum_{j=1}^{\infty} j(j-1) \mathcal{H}(\alpha, \beta, j) a_{j}}{-1-\sum_{j=1}^{\infty} j \mathcal{H}(\alpha, \beta, j) a_{j}}\right)\right] .
\end{aligned}
$$

Thus, by the theorem of maximum modulus, the simple math operation drives the required variation

$$
\sum_{j=1}^{\infty} j[(j+|\tau|)(1-v)+(v-\varepsilon)] \mathcal{H}(\alpha, \beta, j) a_{j} \leq(1-v)(1+|\tau|)+(\varepsilon-v),
$$

This completes the proof.

Theorem (1) immediately gives the next result.

Corollary (1): Let $f \in \mathcal{K}(\varepsilon, \tau, \alpha, \beta, v)$. Then

$$
a_{j} \leq \frac{(1-v)(1+|\tau|)+(\varepsilon-v)}{j[(j+|\tau|)(1-v)+(v-\varepsilon)] \mathcal{H}(\alpha, \beta, j)}, \quad j=1,2, \ldots .
$$

The equality in (9) is obtained for the function $f$ given by (7).

\section{3- Distortion and Growth Theorem}

We now mention the following distortion and growth of class $K(\varepsilon, \tau, \alpha, \beta, v)$.

Theorem (2): Let the function $f \in \mathcal{K}(\varepsilon, \tau, \alpha, \beta, v)$. Then

$\frac{1}{|\mathrm{z}|}-\frac{(1-v)(1+|\tau|)+(\varepsilon-v)}{[(1+|\tau|)(1-v)+(v-\varepsilon)] \mathcal{H}(\alpha, \beta, 1)}|\mathrm{z}| \leq|f(\mathrm{z})| \leq \frac{1}{|\mathrm{z}|}+\frac{(1-v)(1+|\tau|)+(\varepsilon-v)}{[(1+|\tau|)(1-v)+(v-\varepsilon)] \mathcal{H}(\alpha, \beta, 1)}|\mathrm{z}|$.

The outcome is sharp for the function

Proof: Of the function

$$
f(z)=z^{-1}-\frac{(1-v)(1+|\tau|)+(\varepsilon-v)}{[(1+|\tau|)(1-v)+(v-\varepsilon)] \mathcal{H}(\alpha, \beta, 1)} \mathrm{z} .
$$

Likewise

$$
\begin{gathered}
f(\mathrm{z})=\mathrm{z}^{-1}-\sum_{j=1}^{\infty} a_{j} \mathrm{z}^{j} \\
|f(\mathrm{z})| \leq \frac{1}{|\mathrm{z}|}+\sum_{j=1}^{\infty} a_{j}|\mathrm{z}|^{j} \leq \frac{1}{|\mathrm{z}|}+\frac{(1-v)(1+|\tau|)+(\varepsilon-v)}{[(1+|\tau|)(1-v)+(v-\varepsilon)] \mathcal{H}(\alpha, \beta, 1)}|\mathrm{z}| .
\end{gathered}
$$

$$
|f(\mathrm{z})| \geq \frac{1}{|\mathrm{z}|}-\sum_{j=1}^{\infty} a_{j}|\mathrm{z}|^{j} \geq \frac{1}{|\mathrm{z}|}-\frac{(1-v)(1+|\tau|)+(\varepsilon-v)}{[(1+|\tau|)(1-v)+(v-\varepsilon)] \mathcal{H}(\alpha, \beta, 1)}|\mathrm{z}| .
$$

Plural (2.11) and (2.12), we get (2.10).

Theorem (3): Let the function $f \in \mathcal{K}(\varepsilon, \tau, \alpha, \beta, v)$. Then 


$$
\begin{gathered}
\frac{1}{|\mathrm{z}|^{2}}-\frac{(1-v)(1+|\tau|)+(\varepsilon-v)}{[(1+|\tau|)(1-v)+(v-\varepsilon)] \mathcal{H}(\alpha, \beta, 1)} \leq\left|f^{\prime}(\mathrm{z})\right| \\
\leq \frac{1}{|\mathrm{z}|^{2}}+\frac{(1-v)(1+|\tau|)+(\varepsilon-v)}{[(1+|\tau|)(1-v)+(v-\varepsilon)] \mathcal{H}(\alpha, \beta, 1)} . \quad \text { (13) }
\end{gathered}
$$

The outcome is sharp for the function

Proof: Of the function

$$
f(z)=z^{-1}-\frac{(1-v)(1+|\tau|)+(\varepsilon-v)}{[(1+|\tau|)(1-v)+(v-\varepsilon)] \mathcal{H}(\alpha, \beta, 1)} \mathrm{z} .
$$

$$
\begin{gathered}
f(\mathrm{z})=\mathrm{z}^{-1}-\sum_{j=1}^{\infty} a_{j} \mathrm{z}^{j} \\
\left|f^{\prime}(\mathrm{z})\right| \leq \frac{1}{|\mathrm{z}|^{2}}+\sum_{j=1}^{\infty} j a_{j}|\mathrm{z}|^{j} \leq \frac{1}{|\mathrm{z}|^{2}}+\frac{(1-v)(1+|\tau|)+(\varepsilon-v)}{[(1+|\tau|)(1-v)+(v-\varepsilon)] \mathcal{H}(\alpha, \beta, 1)} .
\end{gathered}
$$

Likewise

$$
|f(\mathrm{z})| \geq \frac{1}{|\mathrm{z}|^{2}}-\sum_{j=1}^{\infty} j a_{j}|\mathrm{z}|^{j} \geq \frac{1}{|\mathrm{z}|^{2}}-\frac{(1-v)(1+|\tau|)+(\varepsilon-v)}{[(1+|\tau|)(1-v)+(v-\varepsilon)] \mathcal{H}(\alpha, \beta, 1)} .
$$

Plural (14) and (15), we get (13).

\section{4- Radius of starlikeness and convexity}

Next, we get the region of univalency; in particular, starlikeness and convexity forthe class $\mathcal{K}(\varepsilon, \tau, \alpha, \beta, v)$.

Theorem (4): Let $f \in \mathrm{K}(\varepsilon, \tau, \alpha, \beta, \mathrm{v})$. Then $f$ is starlike of order $\gamma,(0 \leq \gamma<1)$ in $|\mathrm{z}|<r=$ $r_{1}(\varepsilon, \tau, \alpha, \beta, v, \gamma)$, where

$$
r_{1}(\varepsilon, \tau, \alpha, \beta, v, \gamma)=\inf _{j}\left\{\frac{(1-\gamma) j[(j+|\tau|)(1-v)+(v-\varepsilon)] \mathcal{H}(\alpha, \beta, j)}{(j+2-\gamma)(1-v)(1+|\tau|)+(\varepsilon-v)}\right\}^{\frac{1}{j+1}},
$$

$j=1,2, \ldots$.

is a must for every $|z|$ is sharp for each $j$, with the extreme function of form (7).

Proof: Let $f \in \mathcal{K}(\varepsilon, \tau, \alpha, \beta, v)$, then by Theorem (1)

$$
\sum_{j=1}^{\infty} \frac{j[(j+|\tau|)(1-v)+(v-\varepsilon)]}{(1-v)(1+|\tau|)+(\varepsilon-v)} \mathcal{H}(\alpha, \beta, j) a_{j} \leq 1 .
$$

For $0 \leq \gamma<1$, we need to show that

$$
\left|\frac{z f^{\prime}(z)}{f(z)}+1\right| \leq 1-\gamma
$$

we have to show that

Subsequently

$$
\left|\frac{\mathrm{zf}(\mathrm{z})+f(\mathrm{z})}{f(\mathrm{z})}\right| \leq\left|\frac{-\sum_{j=1}^{\infty}(j+1) a_{j} \mathrm{z}^{j+1}}{1-\sum_{j=1}^{\infty} a_{j} \mathrm{z}^{j+1}}\right| \leq \frac{\sum_{j=1}^{\infty}(j+1) a_{j}|\mathrm{z}|^{j+1}}{1-\sum_{j=1}^{\infty} a_{j} \mathrm{z}^{j+1}} \leq 1-\gamma .
$$

This inequality is enough to consider

$$
\sum_{j=1}^{\infty}\left(\frac{j+2-\gamma}{1-\gamma}\right) a_{j}|\mathrm{z}|^{j+1} \leq 1
$$

Therefore

$$
|\mathrm{z}|^{j+1} \leq \frac{(1-\gamma) j[(j+|\tau|)(1-v)+(v-\varepsilon)] \mathcal{H}(\alpha, \beta, j)}{(j+2-\gamma)(1-v)(1+|\tau|)+(\varepsilon-v)} .
$$

$$
|\mathrm{z}| \leq\left\{\frac{(1-\gamma) j[(j+|\tau|)(1-v)+(v-\varepsilon)] \mathcal{H}(\alpha, \beta, j)}{(j+2-\gamma)(1-v)(1+|\tau|)+(\varepsilon-v)}\right\}^{\frac{1}{j+1}} .
$$

Let $|\mathrm{z}|=\mathrm{r}_{1}(\varepsilon, \tau, \alpha, \beta, v, \gamma)$ in (18), We obtain the radius of the starlikeness, and from it the proof of Theorem (4) is complete. 
Theorem (5): Let $f \in \mathcal{K}(\varepsilon, \tau, \alpha, \beta, v)$. Then, $f$ is convex of order $\gamma,(0 \leq \gamma<1)$ in $|z|<r=$ $r_{2}(\varepsilon, \tau, \alpha, \beta, v, \gamma)$, where

$$
r_{2}(\varepsilon, \tau, \alpha, \beta, v, \gamma)=\inf _{j}\left\{\frac{(1-\gamma)[(j+|\tau|)(1-v)+(v-\varepsilon)] \mathcal{H}(\alpha, \beta, j)}{(j+2-\gamma)(1-v)(1+|\tau|)+(\varepsilon-v)}\right\}^{\frac{1}{j+1}},
$$

$j=1,2, \ldots$.

is obligatory for each $|\mathrm{z}|$ sharp per $j$, with the extreme function of the from (7).

Proof: Let $f \in \mathcal{K}(\varepsilon, \tau, \alpha, \beta, v)$, then by Theorem (1)

$$
\sum_{j=1}^{\infty} \frac{j[(j+|\tau|)(1-v)+(v-\varepsilon)]}{(1-v)(1+|\tau|)+(\varepsilon-v)} \mathcal{H}(\alpha, \beta, j) a_{j} \leq 1 .
$$

For $0 \leq \gamma<1$, we have to show that

$$
\left|\frac{z f^{\prime \prime}(\mathrm{z})}{f^{\prime}(\mathrm{z})}+2\right| \leq 1-\gamma
$$

We need to show that

Hence

$$
\left|\frac{z f^{\prime \prime}(\mathrm{z})+2 f^{\prime}(\mathrm{z})}{f^{\prime}(\mathrm{z})}\right| \leq\left|\frac{-\sum_{k=1}^{\infty} j(j+1) a_{j} \mathrm{z}^{j+1}}{1-\sum_{j=1}^{\infty} j a_{j} \mathrm{z}^{j+1}}\right| \leq \frac{\sum_{j=1}^{\infty} j(j+1) a_{j} \mathrm{z}^{j+1}}{1-\sum_{j=1}^{\infty} j a_{j} \mathrm{z}^{j+1}} \leq 1-\gamma .
$$

This is enough to consider

$$
\sum_{j=1}^{\infty} \frac{j(j+2-\gamma)}{1-\gamma} a_{j}|z|^{j+1} \leq 1
$$

Therefore

$$
|\mathrm{z}|^{j+1} \leq \frac{(1-\gamma)[(j+|\tau|)(1-v)+(v-\varepsilon)] \mathcal{H}(\alpha, \beta, j)}{(j+2-\gamma)(1-v)(1+|\tau|)+(\varepsilon-v)} .
$$

$$
|\mathrm{z}| \leq\left\{\frac{(1-\gamma)[(j+|\tau|)(1-v)+(v-\varepsilon)] \mathcal{H}(\alpha, \beta, j)}{(j+2-\gamma)(1-v)(1+|\tau|)+(\varepsilon-v)}\right\}^{\frac{1}{j+1}} .
$$

By setting $|z|=r_{2}(\varepsilon, \tau, \alpha, \beta, v, \gamma)$ in (2.18), we obtain the radius of convexity, which will finish proving the Theorem (5).

\section{5- Hadamard product}

In the subsidiary Theorem, we get the Hadamard product of the functions $f$ and $g \in \mathcal{K}(\varepsilon, \tau, \alpha, \beta, v)$.

Theorem (6): If

$$
f(\mathrm{z})=\mathrm{z}^{-1}-\sum_{j=1}^{\infty} a_{j} \mathrm{z}^{j} \text { and } \mathrm{g}(\mathrm{z})=\mathrm{z}^{-1}-\sum_{j=1}^{\infty} b_{j} \mathrm{z}^{j}
$$

be in the class $\mathcal{K}(\varepsilon, \tau, \alpha, \beta, v)$, then the Hadamard product of $f$ and g, given by

is in the class $\mathcal{K}(\mu, \tau, \alpha, \beta, v)$, where

$$
(f * g)(z)=z^{-1}-\sum_{j=1}^{\infty} a_{j} b_{j} \mathrm{z}^{j},
$$

$\mu$

$$
\leq \frac{[(1-v)(1+|\tau|)+(\varepsilon-v)]^{2}[(j+|\tau|)(1-v)+v]-j[(j+|\tau|)(1-v)+(v-\varepsilon)]^{2} \mathcal{H}(\alpha, \beta, j)[(1-v)(1+|\tau|)-v]}{[(1-v)(1+|\tau|)+(\varepsilon-v)]^{2}+j[(j+|\tau|)(1-v)+(v-\varepsilon)]^{2} \mathcal{H}(\alpha, \beta, j)} .
$$

Proof: Let $f, \mathrm{~g} \in \mathcal{K}(\varepsilon, \tau, \alpha, \beta, v)$. By Theorem (1), we get

and

$$
\sum_{j=1}^{\infty} \frac{j[(j+|\tau|)(1-v)+(v-\varepsilon)]}{(1-v)(1+|\tau|)+(\varepsilon-v)} \mathcal{H}(\alpha, \beta, j) a_{j} \leq 1
$$

$$
\sum_{j=1}^{\infty} \frac{j[(j+|\tau|)(1-v)+(v-\varepsilon)]}{(1-v)(1+|\tau|)+(\varepsilon-v)} \mathcal{H}(\alpha, \beta, j) b_{j} \leq 1 .
$$

We must find the greatest value $\mu$, such that 
By Cauchy--Schwarzs inequality, we get

$$
\sum_{j=1}^{\infty} \frac{j[(j+|\tau|)(1-v)+(v-\mu)]}{(1-v)(1+|\tau|)+(\mu-v)} \mathcal{H}(\alpha, \beta, j) a_{j} b_{j} \leq 1 .
$$

$$
\sum_{j=1}^{\infty} \frac{j[(j+|\tau|)(1-v)+(v-\varepsilon)]}{(1-v)(1+|\tau|)+(\varepsilon-v)} \mathcal{H}(\alpha, \beta, j) \sqrt{a_{j} b_{j}} \leq 1 .
$$

So,

$$
\text { it suffices }
$$

to

show

that

$$
\frac{j[(j+|\tau|)(1-v)+(v-\mu)]}{(1-v)(1+|\tau|)+(\mu-v)} \mathcal{H}(\alpha, \beta, j) a_{j} b_{j} \leq \frac{j[(j+|\tau|)(1-v)+(v-\varepsilon)]}{(1-v)(1+|\tau|)+(\varepsilon-v)} \mathcal{H}(\alpha, \beta, j) \sqrt{a_{j} b_{j}},
$$
that is

And from (25), we will get

$$
\sqrt{a_{j} b_{j}} \leq \frac{[(j+|\tau|)(1-v)+(v-\varepsilon)][(1-v)(1+|\tau|)+(\mu-v)]}{[(j+|\tau|)(1-v)+(v-\mu)][(1-v)(1+|\tau|)+(\varepsilon-v)]} .
$$

Through (26) and (27), it is sufficient to prove that

$$
\sqrt{a_{j} b_{j}} \leq \frac{(1-v)(1+|\tau|)+(\varepsilon-v)}{j[(j+|\tau|)(1-v)+(v-\varepsilon)] \mathcal{H}(\alpha, \beta, j)} .
$$

$$
\begin{array}{r}
\frac{(1-v)(1+|\tau|)+(\varepsilon-v)}{j[(j+|\tau|)(1-v)+(v-\varepsilon)] \mathcal{H}(\alpha, \beta, j)} \\
\leq \frac{[(j+|\tau|)(1-v)+(v-\varepsilon)][(1-v)(1+|\tau|)+(\mu-v)]}{[(j+|\tau|)(1-v)+(v-\mu)][(1-v)(1+|\tau|)+(\varepsilon-v)]}
\end{array}
$$

To simplify that, we have

$$
\leq \frac{[(1-v)(1+|\tau|)+(\varepsilon-v)]^{2}[(j+|\tau|)(1-v)+v]-j[(j+|\tau|)(1-v)+(v-\varepsilon)]^{2} \mathcal{H}(\alpha, \beta, j)[(1-v)(1+|\tau|)-v]}{[(1-v)(1+|\tau|)+(\varepsilon-v)]^{2}+j[(j+|\tau|)(1-v)+(v-\varepsilon)]^{2} \mathcal{H}(\alpha, \beta, j)} .
$$

\section{6- Convex combination}

In the theorems below, we will show that the class $K(\varepsilon, \tau, \alpha, \beta, v)$ is closed under a convex linear combination.

Theorem (7): Let $f_{i} \in \mathcal{K}(\varepsilon, \tau, \alpha, \beta, v)$, such that

Then

$$
f_{i}(\mathrm{z})=\mathrm{z}^{-1}-\sum_{j=1}^{\infty} a_{j, i} \mathrm{z}^{j},\left(a_{j, i} \geq 0, i=1,2\right) .
$$

Also in class $\mathcal{K}(\varepsilon, \tau, \alpha, \beta, v)$.

$$
\varpi(\mathrm{z})=\zeta f_{1}(\mathrm{z})+(1-\zeta) f_{2}(\mathrm{z}), \quad(0 \leq \zeta \leq 1)
$$

Proof: From $0 \leq \zeta \leq 1$, we get

We note that

$$
\varpi(\mathrm{z})=\mathrm{z}^{-1}-\sum_{j=1}^{\infty}\left(\zeta a_{j, 1}+(1-\zeta) a_{j, 2}\right) \mathrm{z}^{j},
$$

$$
\begin{aligned}
& \sum_{j=1}^{\infty} j[(j+|b|)(1-v)+(v-\varepsilon)] \mathcal{H}(\alpha, \beta, j)\left(\zeta a_{j, 1}+(1-\zeta) a_{j, 2}\right) \\
& =\zeta \sum_{j=1}^{\infty} j[(j+|b|)(1-v)+(v-\varepsilon)] \mathcal{H}(\alpha, \beta, j) a_{j, 1} \\
& +(1-\zeta) \sum_{j=1}^{\infty} j[(j+|b|)(1-v)+(v-\varepsilon)] \mathcal{H}(\alpha, \beta, j) a_{j, 2} \leq(1-v)(1+|b|)+(\varepsilon-v) .
\end{aligned}
$$

Using Theorem (1), $\varpi \in \mathcal{K}(\varepsilon, \tau, \alpha, \beta, v)$.

\section{7- Closure theorem}

In the following, we prove the closure theorem.

Theorem (8): Let 


$$
f_{i}(\mathrm{z})=\mathrm{z}^{-1}-\sum_{j=1}^{\infty} a_{j, i} \mathrm{z}^{j} \in \mathcal{K}(\varepsilon, \tau, \alpha, \beta, v), \quad i \in\{1,2, \ldots, m\} \text { and } 0<\vartheta_{i}<1
$$

So that

Then, we select function $\mathcal{G}$ which is defined as

$$
\sum_{i=1}^{m} \vartheta_{i}=1
$$

also in class $\mathcal{K}(\varepsilon, \tau, \alpha, \beta, v)$.

$$
\mathcal{G}(\mathrm{z})=\sum_{i=1}^{m} \vartheta_{i} f_{i}(\mathrm{z})
$$

Proof: For each $i \in\{1,2, \ldots, m\}$, we have

since

$$
\sum_{j=1}^{\infty} \frac{j[(j+|b|)(1-v)+(v-\varepsilon)]}{(1-v)(1+|b|)+(\varepsilon-v)} \mathcal{H}(\alpha, \beta, j) a_{j, i} \leq 1,
$$

And therefore,

$$
\mathcal{G}(\mathrm{z})=\sum_{i=1}^{m} \vartheta_{i} f_{i}(\mathrm{z})=\sum_{i=1}^{m} \vartheta_{i}\left(\mathrm{z}^{-1}-\sum_{j=1}^{\infty} a_{j, i} \mathrm{z}^{j}\right)=\mathrm{z}^{-1}-\sum_{j=1}^{\infty}\left(\sum_{i=1}^{m} \vartheta_{i} a_{j, i}\right) \mathrm{z}^{j} .
$$

$$
\begin{aligned}
& \sum_{j=1}^{\infty} \frac{j[(j+|b|)(1-v)+(v-\varepsilon)]}{(1-v)(1+|b|)+(\varepsilon-v)} \mathcal{H}(\alpha, \beta, j)\left(\sum_{i=1}^{m} \vartheta_{i} a_{j, i}\right) \\
= & \sum_{i=1}^{m} \vartheta_{i}\left(\sum_{j=1}^{\infty} \frac{j[(j+|b|)(1-v)+(v-\varepsilon)]}{(1-v)(1+|b|)+(\varepsilon-v)} \mathcal{H}(\alpha, \beta, j) a_{j, i}\right) \\
\leq \sum_{i=1}^{m} \vartheta_{i}=1 . &
\end{aligned}
$$

Then, $\mathcal{G} \in \mathcal{K}(\varepsilon, \tau, \alpha, \beta, v)$ and the proof is complete.

\section{8- Integral Operators}

Theorem (2.2.8): Let $f \in \mathcal{K}(\varepsilon, \tau, \alpha, \beta, v)$. Then the integral operator [2] defined by

$$
\mathcal{F}(\mathrm{z})=\mathrm{t} \int_{0}^{1} \mathrm{n}^{\mathrm{t}} \mathrm{f}\left(\mathrm{n}_{0} \mathrm{z}\right) \mathrm{dn},\left(0<\mathrm{n}_{0} \leq 1,0<\mathrm{t}<\infty\right),
$$

is also in the class $\mathcal{K}(\varphi, \tau, \alpha, \beta, v)$, such that

$$
\leq \frac{\mathrm{t}[(j+|\tau|)(1-v)+v][(1-v)(1+|\tau|)+(\varepsilon-v)]-[(j+|\tau|)(1-v)+(v-\varepsilon)][(j+\mathrm{t}+1)(1-v)(1+|\tau|)-v]}{\mathrm{t}[(1-v)(1+|\tau|)+(\varepsilon-v)]+[(j+|\tau|)(1-v)+(v-\varepsilon)]} .
$$

Proof: Let $f \in \mathcal{K}(\varepsilon, \tau, \alpha, \beta, v)$, and therefore we have

We suffice it to prove that

$$
\begin{aligned}
\mathcal{F}(\mathrm{z})=\mathrm{t} \int_{0}^{1} \mathrm{n}_{0}^{\mathrm{t}} f\left(\mathrm{n}_{0} \mathrm{z}\right) \mathrm{dn}, & =\mathrm{t} \int_{0}^{1}\left(\mathrm{n}^{\mathrm{t}-1} \mathrm{z}^{-1}-\sum_{j=1}^{\infty} \mathrm{n}^{j+\mathrm{t}} a_{j} \mathrm{z}^{j}\right) d \mathrm{n}_{0} \\
& =\mathrm{z}^{-1}-\sum_{j=1}^{\infty} \frac{\mathrm{t}}{j+\mathrm{t}+1} a_{j} \mathrm{z}^{j} .
\end{aligned}
$$

$$
\sum_{j=1}^{\infty} \frac{\mathrm{t}_{j}[(j+|\tau|)(1-v)+(v-\varphi)]}{(j+t+1)(1-v)(1+|\tau|)+(\varphi-v)} \mathcal{H}(\alpha, \beta, j) a_{j} \leq 1,
$$

since the function $f \in \mathcal{K}(\varepsilon, \tau, \alpha, \beta, v)$, then we will get

$$
\sum_{j=1}^{\infty} \frac{j[(j+|\tau|)(1-v)+(v-\varepsilon)]}{(1-v)(1+|\tau|)+(\varepsilon-v)} \mathcal{H}(\alpha, \beta, j) a_{j} \leq 1 .
$$


Note that (29) is satisfied if the following inequality is satisfied

$$
\frac{\mathrm{t}_{j}[(j+|\tau|)(1-v)+(v-\varphi)]}{(j+\mathrm{t}+1)(1-v)(1+|\tau|)+(\varphi-v)} \mathcal{H}(\alpha, \beta, j) \leq \frac{j[(j+|\tau|)(1-v)+(v-\varepsilon)]}{(1-v)(1+|\tau|)+(\varepsilon-v)} \mathcal{H}(\alpha, \beta, j) .
$$

And by rewriting the inequality, we will get

$$
\begin{aligned}
& \mathrm{t}[(j+|\tau|)(1-v)+(v-\varphi)][(1-v)(1+|\tau|)+(\varepsilon-v)] \\
& \leq[(j+t+1)(1-v)(1+|\tau|)+(\varphi-v)][(j+|\tau|)(1-v)+(v-\varepsilon)]
\end{aligned}
$$

As a solution to $\varphi$, we will get

$$
\leq \frac{\mathrm{t}[(j+|\tau|)(1-v)+v][(1-v)(1+|\tau|)+(\varepsilon-v)]-[(j+|\tau|)(1-v)+(v-\varepsilon)][(j+\hbar+1)(1-v)(1+|\tau|)-v]}{\mathrm{t}[(1-v)(1+|\tau|)+(\varepsilon-v)]+[(j+|\tau|)(1-v)+(v-\varepsilon)]} .
$$

Thus, the right side of (30) is an increasing function of $j$.

\section{Neighborhoods}

Following earlier investigations on the familiar meaning of neighborhoods of meromorphic functions, which was first introduced by [9], a later work [10] studies this concept with elements of many famous subclasses of analytic functions. An earlier work [11] considered a specific family of meromorphic functions with negative coefficients. Also, other studies [2, 12, 13, 14] extended this topic for a specific subclass of meromorphically multivalent functions or meromorphically univalent functions.

The $(j, \sigma)$ - neighborhood of a function $f \in A(j)$ is defined by the following relation:

$$
N_{j, \sigma}(f)=\left\{\mathrm{g} \in \mathcal{A}(j): \mathrm{g}(\mathrm{z})=\mathrm{z}^{-1}-\sum_{j=1}^{\infty} b_{j} \mathrm{z}^{j} \text { and } \sum_{j=1}^{\infty} j\left|a_{j}-b_{j}\right| \leq \sigma, 0 \leq \sigma<1\right\} .
$$

For the identity function $\mathrm{e}(\mathrm{z})=\mathrm{z}$, we will get

$$
N_{j, \sigma}(e)=\left\{\mathrm{g} \in \mathcal{A}(j): \mathrm{g}(\mathrm{z})=\mathrm{z}^{-1}-\sum_{j=1}^{\infty} b_{j} \mathrm{z}^{j} \text { and } \sum_{j=1}^{\infty} j\left|b_{j}\right| \leq \sigma\right\} .
$$

Definition (2): A function $f \in \mathcal{A}(j)$ defined by definition (1) is said to be in the class $\mathcal{K}^{\rho}(\varepsilon, \tau, \alpha, \beta, v)$ if there is a function $\mathrm{g} \in \mathcal{K}(\varepsilon, \tau, \alpha, \beta, v)$ such that

$$
\left|\frac{f(\mathrm{z})}{\mathrm{g}(\mathrm{z})}-1\right|<1-\rho, \quad(\mathrm{z} \in U, 0 \leq \rho<1) .
$$

Theorem (10): Let $\mathrm{g} \in \mathcal{K}^{\rho}(\varepsilon, \tau, \alpha, \beta, \mathrm{v})$ and

$$
\rho=1-\frac{\sigma[(1+|\tau|)(1-v)+(v-\varepsilon)] \mathcal{H}(\alpha, \beta, 1)}{[(1+|\tau|)(1-v)+(v-\varepsilon)] \mathcal{H}(\alpha, \beta, 1)-[(1-v)(1+|\tau|)+(\varepsilon-v)]} .
$$

Then, $\quad \mathrm{N}_{j, \sigma}(\mathrm{g}) \subset \mathcal{K}^{\rho}(\varepsilon, \tau, \alpha, \beta, \mathrm{v})$.

Proof: Let $f \in N_{j, \sigma}(\mathrm{g})$. Then, we will get from (31) that

which denotes the coefficient inequality

$$
\sum_{j=1}^{\infty} j\left|a_{j}-b_{j}\right| \leq \sigma,
$$

$$
\sum_{j=1}^{\infty}\left|a_{j}-b_{j}\right| \leq \sigma,(j \in \mathbb{N})
$$

Since the function

$$
\begin{aligned}
g & \in \mathcal{K}(\varepsilon, \tau, \alpha, \beta, v), \text { then we get from } \\
\sum_{j=1}^{\infty} b_{j} & \leq \frac{(1-v)(1+|\tau|)+(\varepsilon-v)}{[(1+|\tau|)(1-v)+(v-\varepsilon)] \mathcal{H}(\alpha, \beta, 1)},
\end{aligned}
$$

and, hence, it is

$$
\begin{gathered}
\left|\frac{f(\mathrm{z})}{\mathrm{g}(\mathrm{z})}-1\right| \leq \frac{\sum_{j=1}^{\infty}\left|a_{j}-b_{j}\right|}{1-\sum_{j=1}^{\infty} b_{j}} \\
\leq \frac{\sigma[(1+|\tau|)(1-v)+(v-\varepsilon)] \mathcal{H}(\alpha, \beta, 1)}{[(1+|\tau|)(1-v)+(v-\varepsilon)] \mathcal{H}(\alpha, \beta, 1)-[(1-v)(1+|\tau|)+(\varepsilon-v)]}=1-\rho .
\end{gathered}
$$

Therefore, by using definition (2), $f \in \mathcal{K}^{\rho}(\varepsilon, \tau, \alpha, \beta, v)$ for $\sigma$ given by equation (33). 


\section{References}

1. Duren, P. T. 1983. Univalent Functions. Grundleheren der Mathematischen Wissenschaften 259, Springer-Verlag, New York, Berlin, Heidelberg, Tokyo, (1983).

2. Atshan, W. G. 2008. Subclass of meromorphic functions with positive coefficients defined by Ruscheweyh derivative II. Surveys in Mathematics and its Applications, 3: 67-77.

3. Atshan, W. G. and Kulkarni, S. R. 2007, On a class of p-valent meromorphic functions defined by integral operator. International J. Math. Sci. \& Engg. Appls. (IJMSEA), 1(1): 129-140.

4. Clunie, J. G.1959. On meromorphic schlicht functions. J. London Math. Soc. , 34: 215-216.

5. Lafta, M. H. and AL- Zubaidy, K. A. 2017. On a New class of Meromorphically Univalent Functions with Applications to Geometric Functions. Engineering \&Technology Journal, 35(2): 215-220.

6. Lafta, M. H. and AL- Zubaidy, K. A. 2018. Some Geometric Properties of Multivalent Functions Defined on Hilbert Space. Iraqi Journal of Science., 59(12): 564- 671.

7. Miller, J. E. 1970. Convex meromorphic mapping and related functions. Proc. Amer. Math. Soc., 25: $220-228$.

8. Orhan, H. Răducanu, D. and Deniz, E. 2010. Subclass of meromorphically multivalent functions defined by differential operator. Math. CV. 27: 1-23.

9. Goodman, A. W. 1957. Univalent functions and non-analytic curve. Proc. Amer. Math. Soc., 8: 598-601.

10. Ruscheweyh, S. 1981. Neighborhoods of univalent functions. Proc. Amer. Math. Soc. , 81: 521527.

11. Altintas, O. and Owa, S. 1996. Neighborhoods of certain analytic functions with negative coefficients. Int. J. Math. Sci., 19: 797-800.

12. Atshan, W. G. and Joudah, A. S. 2011, Subclass of meromorphic univalent functions defined by Hadamard product with multiplier transformations. International Mathematical Forum, 6(46): 2279-2292.

13. Atshan, W. G. and Sulman, J. H. 2011. On a class of meromorphic univalent functions defined by linear derivative operator. International Mathematical Forum, 6(46): 2267-2278.

14. Liu, J. -L. and Srivastava, H. M. 2001. A linear operator and associated families of meromorphically multivalent functions. J. Math. Anal. Appl. 259: 566-581. 